\title{
Long-term health and germline transmission in transgenic cattle following transposon- mediated gene transfer
}

Soo-Young Yum ${ }^{1 \dagger}$, Song-Jeon Lee ${ }^{2 \dagger}$, Sin-Gi Park ${ }^{3}$, In-Gang Shin ${ }^{3}$, Sang-Eun Hahn ${ }^{1}$, Woo-Jae Choi ${ }^{1}$, Hee-Soo Kim², Hyeong-Jong Kim², Seong-Hun Bae², Je-Hyeong Lee², Joo-Yeong Moon², Woo-Sung Lee², Ji-Hyun Lee', Choong-II Lee ${ }^{1}$, Seong-Jin Kim ${ }^{3}$ and Goo Jang ${ }^{1,4^{*}}$

\begin{abstract}
Background: Transposon-mediated, non-viral gene delivery is a powerful tool for generating stable cell lines and transgenic animals. However, as multi-copy insertion is the preferred integration pattern, there is the potential for uncontrolled changes in endogenous gene expression and detrimental effects in cells or animals. Our group has previously reported on the generation of several transgenic cattle by using microinjection of the Sleeping Beauty (SB) and PiggyBac (PB) transposons and seeks to explore the long-term effects of this technology on cattle.

Results: Transgenic cattle, one female (SNU-SB-1) and one male (SNU-PB-1), reached over 36 months of age with no significant health issues and normal blood parameters. The detection of transgene integration and fluorescent signal in oocytes and sperm suggested the capacity for germline transmission in both of the founder animals. After natural breeding, the founder transgenic cow delivered a male calf and secreted milk containing fluorescent transgenic proteins. The calf expressed green fluorescent protein in primary cells from ear skin, with no significant change in overall genomic stability and blood parameters. Three sites of transgene integration were identified by next-generation sequencing of the calf's genome.
\end{abstract}

Conclusions: Overall, these data demonstrate that transposon-mediated transgenesis can be applied to cattle without being detrimental to their long-term genomic stability or general health. We further suggest that this technology may be usefully applied in other fields, such as the generation of transgenic animal models.

Keywords: Germline transmission, Next-generation sequencing, Sleeping beauty, PiggyBac, Transgenic cattle

\section{Background}

Transposon-mediated gene delivery is a valuable technique for use in gene therapy and ex vivo gene delivery and for in vitro cell line and animal model generations [1-4]. Of the several DNA transposons available, Sleeping Beauty (SB) and PiggyBac (PB) transposons have been the most widely used to deliver exogenous genes into cell lines and to generate transgenic animals. In

\footnotetext{
* Correspondence: snujang@snu.ac.kr

${ }^{\dagger}$ Soo-Young Yum and Song-Jeon Lee contributed equally to this work. 'Department of Theriogenology, College of Veterinary Medicine and the Research Institute of Veterinary Science, Seoul National University, \#631 Building 85, Gwanak-ro, Gwanak-gu, Seoul 08826, Republic of Korea ${ }^{4}$ Emergence Center for Food-Medicine Personalized Therapy System, Advanced Institutes of Convergence Technology, Seoul National University, Gyeonggi-do 16229, Republic of Korea

Full list of author information is available at the end of the article
}

mice and rats, it is well established that transposonmediated transgenesis can result in germline transmission to create transgenic offspring. Transposons such as SB and PB have also been used successfully to produce transgenic pigs, sheep, goats and cattle [5-10], and in the case of the transgenic pigs, germline transmission was also observed [11].

The transposon system is particularly valuable as it mediates both the efficient genomic integration and stable expression of transgenes in target cells or animals. The transposase enzyme binds specifically to the recognition sequences of the transposon and induces the 'cutand-paste' integration of a transposon vector into the genome in a site-specific manner (SB integrates at TA sequences and $\mathrm{PB}$ integrates at TTAA) free from vector

(c) The Author(s). 2018 Open Access This article is distributed under the terms of the Creative Commons Attribution 4.0 International License (http://creativecommons.org/licenses/by/4.0/), which permits unrestricted use, distribution, and 
backbone DNA [12]. This system can be used in conjunction with various non-viral delivery systems, such as chemical-based transfection, electroporation or microinjection. In comparison to viral delivery and transgene integration systems, transposon systems are more secure and safe to use $[13,14]$ and this has led to NIH-OBA and FDA approval for testing of the SB transposon in humans [15-17].

In vitro studies have shown that the random nature of $\mathrm{SB}$ and PB insertion events can lead to insertional mutagenesis [4, 18]. However, as transposon-mediated gene transfers can also integrate within non-coding regions, the system is still considered far safer than viral delivery systems in terms of potential geno-toxicity. In support of this, our study on transgene integration sites in transgenic cattle demonstrated that all transgenes were integrated within non-coding and non-functional regions [5]. However, such genomic analyses may not be entirely predictive of general health in transgenic animals. Although transgenic cattle have been generated via transposon-, viral vector- or somatic cell nuclear transfer-mediated gene transfers, to date there has been no report on long-term monitoring of health issues in such cattle or their offspring. Therefore, we have sought to test our hypothesis that multi-copy transgene integration in transgenic cattle (founders) will not affect their long-term survival (over 3 years) and, further, will undergo germline transmission.

\section{Methods}

\section{Animals}

All the transgenic cattle involved in this study were reported in our previous publication [5]. Briefly, the transgenic cattle (females SNU-SB-1 and SNU-PB-2, and male SNU-PB-1) were derived from embryos which had undergone microinjection in order to introduce either of the two transposon systems (SB or PB) as indicated.

\section{Cell isolation and culture}

Primary cultures of fibroblasts were derived from ear skin biopsies of transgenic cattle. The ear skin was cut into pieces of $1-4 \mathrm{~mm}$ in size with a sterile scalpel before being washed several times and incubated at $37^{\circ} \mathrm{C}$ for $16 \mathrm{~h}$ in HBSS supplemented with collagenase (Collagenase type IV, Gibco). The dispersed cells were washed in HBSS and cultured in DMEM supplemented with antibiotics and $10 \%$ fetal calf serum.

\section{Semen collection and freezing}

Semen from the male transgenic founder was collected using an artificial vagina (Fujihira Industry, Tokyo, Japan) containing warm water at $50-55{ }^{\circ} \mathrm{C}$. The collected semen was immediately transported to the laboratory for freezing. The semen was diluted 1:1 with OPTIXcell (IVM technologies, France) and incubated at room temperature for $10 \mathrm{~min}$. The semen was further diluted 1:1 to a sperm concentration of around $5.0 \times 10^{7}$ cells $/ \mathrm{mL}$ before incubation at $4{ }^{\circ} \mathrm{C}$ for $2 \mathrm{~h}$. The concentrated sperm solution was loaded into a $500 \mu \mathrm{L}$ semen straw (IMV technologies, France) and sealed with straw powder (Fujihira Industry, Tokyo, Japan). The straw underwent freezing $5.0 \mathrm{~cm}$ above the surface of liquid nitrogen for $30 \mathrm{~min}$ and was then plunged into a liquid nitrogen tank for storage.

\section{In vitro oocyte maturation, fertilization and culture of embryos}

Ovaries were obtained from a local abattoir and maintained in saline at $35^{\circ} \mathrm{C}$ during transport to the laboratory. Cumulus-oocyte complexes (COCs) from follicles 2 to $8 \mathrm{~mm}$ in diameter were aspirated using an 18-guage needle, selected and collected in a conical tube. The sediment was washed three times with HEPES-buffered tissue culture medium-199 (TCM-199; Invitrogen, Carlsbad, CA, USA) supplemented with $2 \mathrm{mM} \mathrm{NaHCO} 3$ (SigmaAldrich Corp., St. Louis, MO, USA), 10\% FBS and 1\% penicillin-streptomycin $(v / \mathrm{v})$. For in vitro maturation, COCs were cultured for $22 \mathrm{~h}$ in $450 \mu \mathrm{L}$ TCM-199 supplemented with $0.005 \mathrm{AU} / \mathrm{mL}$ FSH (Sigma-Aldrich), 10\% FBS, $1 \mu \mathrm{g} / \mathrm{mL}$ 17ß-estradiol (Sigma-Aldrich) and $100 \mu \mathrm{M}$ Cysteamine (Sigma-Aldrich) at $39^{\circ} \mathrm{C}$ under $5 \% \mathrm{CO}_{2}$.

The Percoll gradient method for the separation and purification of motile spermatozoa has been described in detail elsewhere [19]. Briefly, spermatozoa were purified from thawed semen straws by density-gradient centrifugation on a Percoll discontinuous gradient $(45-90 \%)$ at $1500 \mathrm{rpm}$ for $15 \mathrm{~min}$. The Percoll density gradient was prepared by layering $1 \mathrm{~mL}$ of $45 \%$ Percoll solution onto $1 \mathrm{~mL}$ of $90 \%$ Percoll solution in a $15 \mathrm{~mL}$ conical tube. The thawed semen was layered onto the top of the Percoll gradient solution and the tube was centrifuged. The pellet was washed twice with TALP by centrifugation for $5 \mathrm{~min}$ at $1500 \mathrm{rpm}$. The active, motile spermatozoa from the pellet were added to droplets containing matured oocytes. Oocytes were inseminated on day 0 with $1-2 \times 10^{6}$ spermatozoa/mL for $18 \mathrm{~h}$ in IVF-TALP medium (Nutricell) under mineral oil. The fertilized oocytes were denuded and cultured in two-step defined culture medium ( 5 days in D1 medium before transfer to $\mathrm{D} 2$ medium) at $39^{\circ} \mathrm{C}$ in an atmosphere of $5 \% \mathrm{O}_{2}, 5 \%$ $\mathrm{CO}_{2}$ and $90 \% \mathrm{~N}_{2}[20]$.

\section{Blood analysis and veterinary care}

A veterinarian collected $5 \mathrm{ml}$ whole blood samples from the jugular vein for blood analysis and monitored regularly general health condition. Some were used for Complete Blood Count (CBC) (Hemavet 950, Drew 
Scientific, USA) and the others were used for serum chemistry analysis (BS-400, Mindray, China).

One transgenic cow (SNU-PB-2) was injured by other cattle, leading to severe respiratory distress. Under the advice of veterinarians, we decided to euthanize the cow. The planned method of euthanasia was administration of a general anesthetic reagent before the use of pentobarbital. However, due to severe organ damage caused by the respiratory distress, only the general anesthetic (Xylazine, BAYER; $0.15 \mathrm{mg} / \mathrm{kg}$ intravenous) was administered to be euthanasia.

\section{Library preparation for massively parallel sequencing} Purified genomic DNA from F0 (SNU-SB-1, SNU-PB-1) samples and the SNU-F1-1 sample was randomly sheared using a Covaris S2 Ultrasonicator to yield DNA fragments of on average $350 \mathrm{bp}$ in size. Library preparation was performed using the Illumina TruSeq DNA PCR-free preparation kit. Adaptor enrichments were performed using PCR according to the manufacturer's instructions. The final library size and quality were evaluated by electrophoresis using an Agilent High Sensitivity DNA kit. The 150 bp paired-end reads were sequenced with an Illumina HiSeq 4000 platform. Further image analysis and base calling were performed with RTA 2.7.3 (Real Time Analysis) and bcl2fastq v2.17.1.14.

\section{Read alignment}

Sequenced reads were filtered using sickle (v1.33) with a Phred quality threshold of 20 to derive high-quality reads. The remaining reads were mapped against the Bos taurus genome (UMD 3.1, http://asia.ensembl.org/Bos_ taurus/Info/Annotation) and the transgene sequence simultaneously using BWA ver. 0.7.5a. After mapping, duplicates were marked using Picard ver. 1.128 and local realignment was then performed using GATK ver. 3.6-0.

\section{Variant analysis}

Multi-sample calling (including F0 samples) of single nucleotide variants (SNV) and insertions and deletions (INDELs) was performed using GATK ver.3.6-0 with UnifiedGenotyper. After multi-sample calling, variants were filtered using a genotype quality value cutoff of 60 . The SnpEff software was used together with the UMD3. 1.79 Bos taurus Ensembl annotation set to predict the functional effects of the variants detected.

\section{Assessment of genomic stability}

In order to investigate potential genomic instability, we classified SNPs and INDELs into three groups: RefHom (homozygous reference genotype), Hetero (heterozygous genotype) and AltHom (homozygous alteration genotype). Genetic variants (SNPs and INDELs) were assigned using the following criteria: (1) if the sequence is classified as RefHom, the ratio to the reference allele depth was more than $90 \%$; (2) if the sequence is classified as Hetero, the ratio to the reference allele depth was more than $40 \%$ and less than $60 \%$; (3) if the sequence is classified as AltHom, the ratio to the altered allele depth was more than $90 \%$. We also removed the mitochondrial genome, $\mathrm{X}$ chromosome and unanchored scaffolds from further analysis. Finally, we searched remaining variants as de novo mutations rather than inherited sequences.

\section{Identification of copy number variations (CNVs)}

The Control-FREEC software was applied for the identification of copy number changes in the genomes of the transgenic cattle. The software is used for calculation of the ploidy of regions of interest, with the copy number value calculated for a $50 \mathrm{~kb}$ window in the region of interest, following GC content read count normalization, and compared to a normal autosomal ploidy value of two.

\section{Detection of transgene insertion sites}

Transgene insertion sites were identified some softclipped nucleotide following mapping with BWA. Some soft-clipped nucleotides could be determined by a Smith-Waterman-like scoring scheme in the BWA software. The candidate insertion sites were inferred by inspection of the mapped pattern in the soft-clipped sequences. Delly software was also applied in parallel for estimation of genomic structural variation as an indicator of transgene insertion. Finally, we manually inspected the candidate sites with IGV software.

\section{Calculation of telomere length using the whole genome sequence}

Reads that were rich in telomere sequences were extracted from the whole genome sequence dataset and their relative length determined. We then applied TelSeq software to reveal any difference in the number of copies of TTAGGG between transgenic and control genomes through calculation of the frequency of reads.

\section{Measurement of fluorescence intensity}

To quantify the fluorescence intensities of samples from SNU-F1-1 and SNU-F1-2, images of cells of the same passage and density were acquired. Using ImageJ (v1.50, $\mathrm{NIH}$ ), an equally-sized region was selected using the square-drawing function of the drawing/selection tools and pixel measurements (area, mean gray value and integrated density) acquired from this region of interest. The integrated density for each cell was calculated for the region of interest. 


\section{Results}

\section{Health monitoring using blood analysis}

Both female (SNU-SB-1) and male (SNU-PB-1) transgenic cattle reached ages of 50 and 43 months, respectively, without any health problems (Fig. 1). Analysis of blood parameters (white blood cells, WBC; red blood cells, RBC; platelets) showed no significant difference between the blood of the transgenic animals and that of the reference (Fig. 2). Furthermore, there was no significant difference in various serum chemical parameters, including alanine transaminase (ALT), aspartate transaminase (AST), and blood urea nitrogen $(\mathrm{BUN})$, between the transgenic animals and the reference (Additional file 1).

\section{Germline transmission of the transgene}

To assess the germline transmission in sperm of transgenes introduced by transposons, sperm was harvested from SNU-PB-1 after puberty using manual ejaculation and cryopreserved to create frozen semen stocks. Over 200 straws were produced and preserved in liquid nitrogen for future use. The motility of frozen-thawed semen was normal, and the semen was used for in vitro fertilization (IVF) together with wild-type oocytes. Around $88 \%$ of blastocysts expressed green fluorescent protein (GFP) in every round of IVF undertaken (Fig. 3). Thus, the frozen sperm can be used to rapidly increase the population of transgenic cattle. These results demonstrate that germline transmission of transgenes introduced by transposon systems is possible.

\section{Natural birth of an F1 calf from transgenic cattle}

SNU-SB-1 was mated naturally with SNU-PB-1 in order to assess the stability of germline transmission of the transgene to the resultant offspring. The F1 (SNU-F1-1, male) was delivered without any assistance following the gestation period (Fig. 4b) and a physical examination concluded that there were no congenital defects. Expression of the GFP transgene in the eyes of the calf was apparent without the use of equipment. To further investigate the germline transmission of the transgene, skin fibroblasts were isolated from SNU-F1-1, cultured and expanded for genomic analysis. All of the fibroblasts homogenously expressed GFP (Fig. 4c) and PCR analysis of the genomic DNA from these cells demonstrated the presence of GFP transgenes in the genome (Fig. 4d). The construct integrated into the genome of the SNU-PB-1 father contained rox-flanked (froxed) GFP followed by red fluorescent protein (RFP) [5]. We therefore expected that if the same parental transgenes had been successfully transmitted to SNU-F1-1, the expression of Dre recombinase should excise the froxed GFP from the genome, leaving only a single rox site and RFP in the genome (Fig. 4a). As expected, electroporation of Dre recombinase into cells from SNU-F1-1 was sufficient to induce the expression of RFP and the deletion of froxed GFP was confirmed by PCR analysis (Fig. 4c-d). In contrast, we did not detect transmission of yellow fluorescent protein (YFP) transgenes from the mother (Additional file 2: Figure S1). These data indicate the successful transmission of transgenes from SNU-PB-1 to F1 offspring. Furthermore, the F1 calf SNU-F1-1 was healthy, with no significant abnormalities detected by blood analysis (Fig. 2).

We examined wild-type milk and transgenic milk by confocal microscopy to determine whether the transgenic fluorescent protein would be detected in milk from SNU-SB-1 after delivery of SNU-F1-1. As expected, YFP was observed in the milk from SNU-SB-1 (Additional file 3: Figure S2). This result suggests that transposon-derived transgenic cattle can be used as bioreactors for the production of recombinant proteins.

\begin{tabular}{|c|c|c|c|c|c|}
\hline I.D. & Transposon & Transgene & Copy Number & Gender & Age \\
\hline SNU-SB-1 [5] & Sleeping Beauty & pCAG-YFP & 3 & Female & 50months \\
\hline SNU-PB-1 [5] & Piggybac & pCAG-rox-GFP-rox-RFP & 15 & Male & 43 months \\
\hline
\end{tabular}
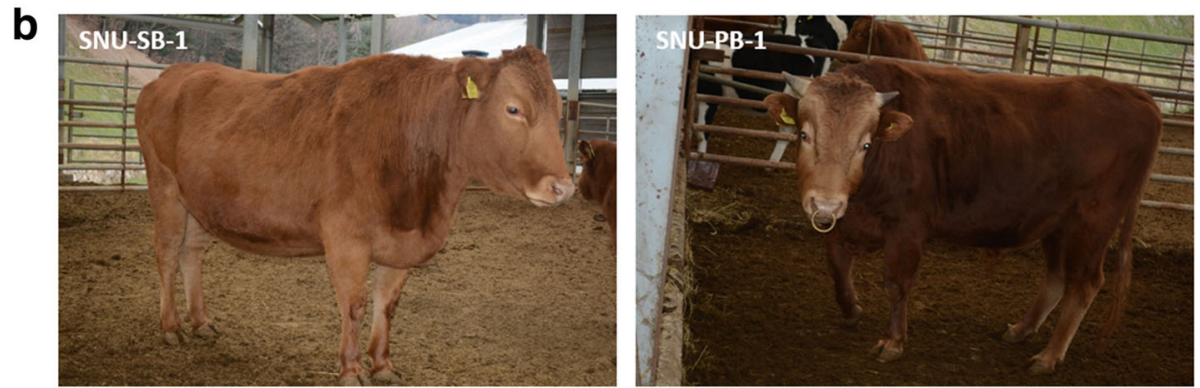

Fig. 1 Overview of transposon-derived transgenic cattle in this study. a Summary of transgene and general information for the transgenic cattle. b Recent images of the transgenic cattle. Left: SNU-SB-1; right: SNU-PB-1 


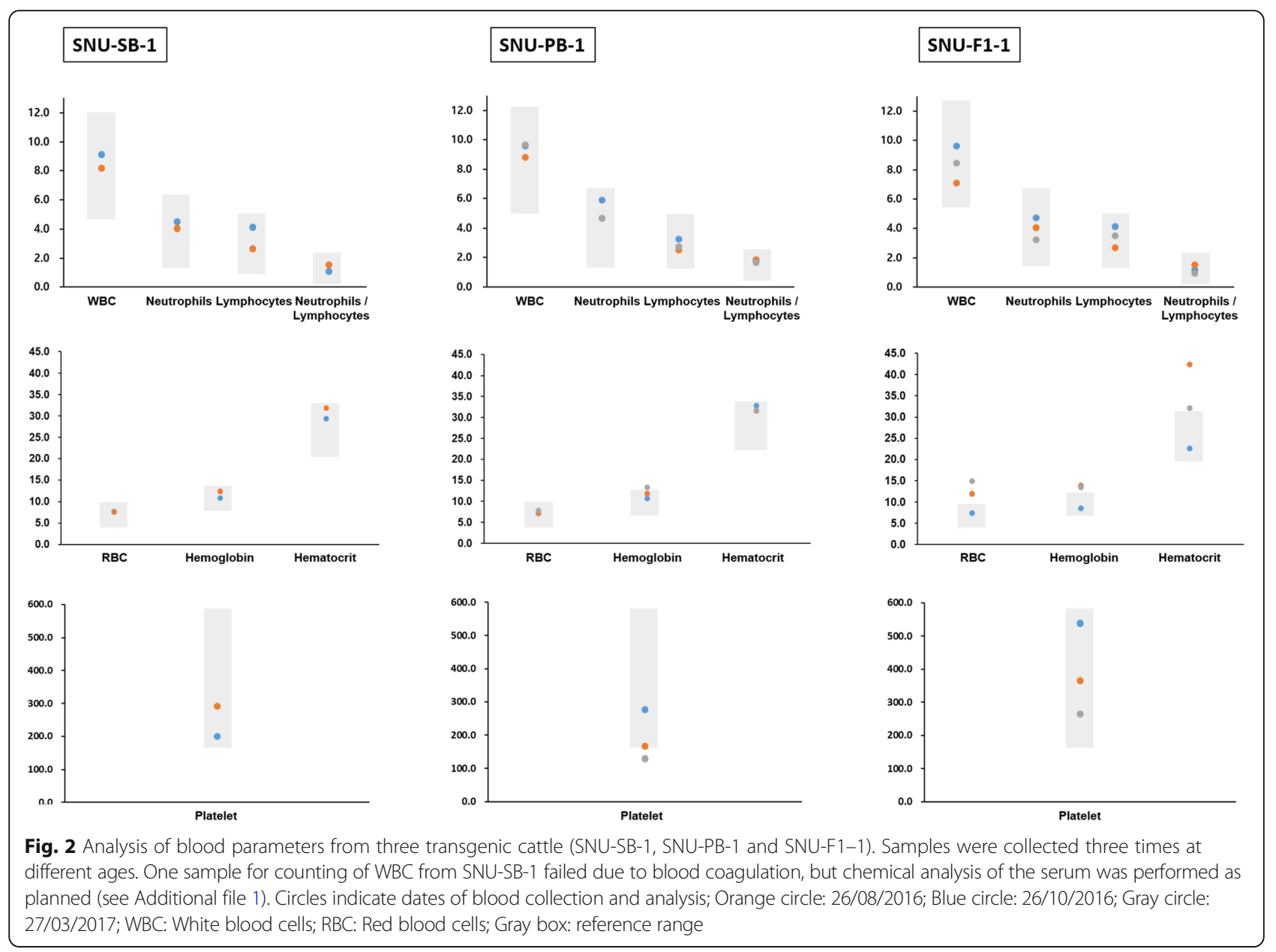

Whole genome sequencing to determine integration sites and genomic stability

To confirm the integration sites of the transgenes within the maternal and paternal genomes, whole genome sequencing was performed with next-generation sequencing (NGS). NGS has been widely used for highthroughput genomic analysis, such as molecular characterization and structure variation. We confirmed the transgene insertion sites previously identified in the
F0 transgenic cattle $[5,21]$. NGS of the SNU-F1-1 genome was performed to determine the transgene insertion sites. The sequencing showed that a total of three copies of the paternal transgene were transmitted to the F1 calf, and we determined the sites of integration for the transgenes to be in non-coding regions (Table 1). Transgene insertion sites were verified by $5^{\prime}$ junction sequence analysis using a specific primer set that anneals to the unique genome-to-transposon junction in chromosomes

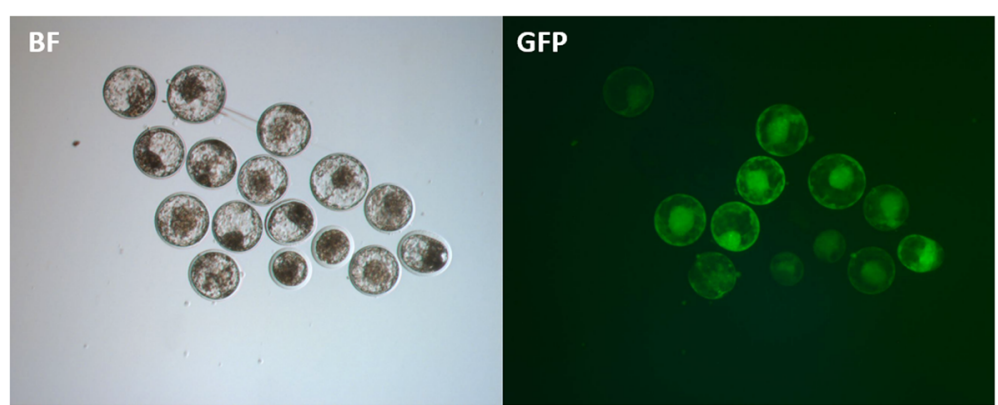

Fig. 3 Germline transmission of transgenes in spermatozoa from SNU-PB-1. Blastocyst stage embryos express GFP after IVF using frozen-thawed semen from SNU-PB-1. BF: bright field; GFP: GFP field 
a

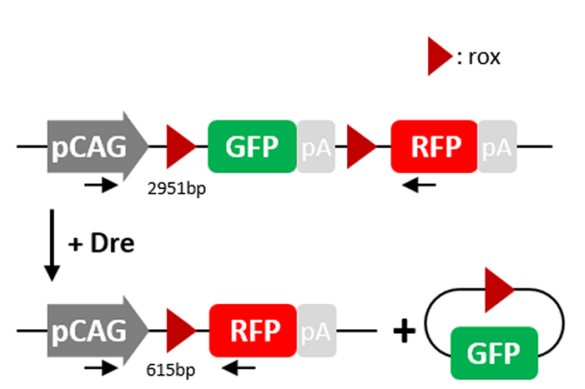

C

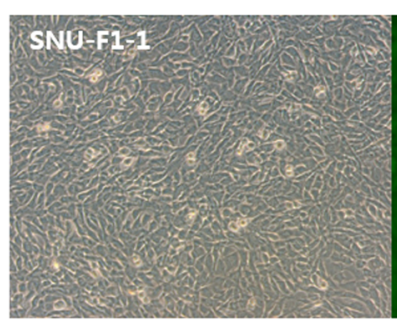

b

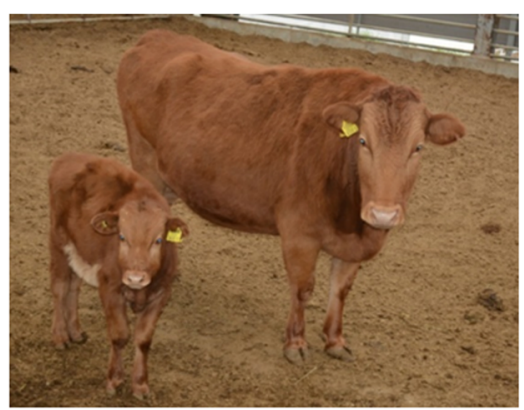

GFP

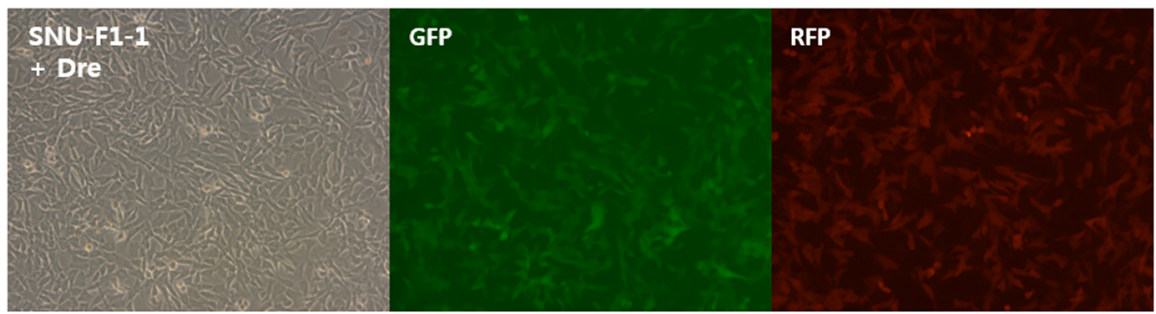

d

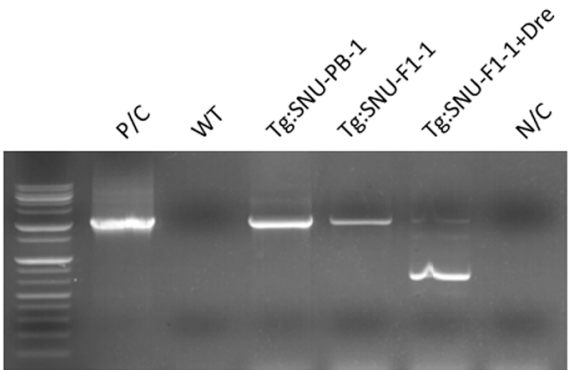

Fig. 4 Detection of transgene expression in SNU-F1-1. a Schematic of Dre-rox recombination in constructs used in this study. $\mathbf{b}$ Image of SNUF1-1 (left) and its mother, SNU-SB-1 (right). c Primary cells from the ear skin of SNU-F1-1 express the transgenic reporter protein, GFP (upper). RFP expression is detected following transfection of ear skin cells with Dre recombinase (lower). $\mathbf{d}$ PCR analysis of Dre-rox recombination using genomic DNA from the cells derived from SNU-F1-1. P/C, positive control (PB-CA-Rox-GFP-Rox-RFP vector); WT, genomic DNA from wild type cattle; Tg:SNU-PB-1, genomic DNA from the blood of SNU-PB-1; Tg:SNU-F1-1, genomic DNA from the blood of SNU-F1-1; Tg:SNU-F1-1 + Dre, genomic DNA from cells from SNU-F1-1 which have undergone transfection with Dre recombinase; N/C, negative control (nuclease-free water)

Table 1 All integration sites in SNU-F1-1

\begin{tabular}{llllll}
\hline No. & Chromosome & Insertion site & Orientation & 5' gene & $3^{\prime}$ gene \\
\hline 1 & 4 & $95,433,564-95,434,563$ & Forward & TSGA13 & MKLN1 \\
2 & 4 & $113,823,097-113,823,101$ & Forward & ENSBTAG00000001198.5 & ENSBTAG00000046257.1 \\
3 & 6 & $20,085,913-20,086,912$ & Forward & DKK2 & GIMD1 \\
\hline
\end{tabular}


4 (two sites) and 6 (Additional file 4: Figure S3). Additionally, we compared the genome of SNU-F1-1 to those of its parents (F0 samples) and identified 147 heterozygous de novo mutations and 2 homozygous de novo mutations differing from the maternal and paternal genomes (Table 2). The heterozygous de novo mutations were classified by position into intergenic $(125,78.61 \%)$, intronic $(33,20.76 \%)$ and exonic $(1,0.63 \%$, in the ENSBTAG00000038261 gene) mutations, with most occurring in the intergenic and intronic regions. In addition, the homozygous de novo mutations were identified mistaken searched variants because they occurred in the long terminal repeat (LTR) region. The number of de novo mutations is consistent with previous studies [22], corresponding to a mutation rate of $5.62 \times 10^{-8}$ per position per generation per genome. And then, we identified the telomere length is 10.23. We have summarized the SNVs, INDELs and CNVs detected (Fig. 5).

\section{Correlation of GFP expression level with transgene copy number}

There are several studies which demonstrate a correlation between the level of protein expression and transgene copy number [23, 24]. To investigate the relationship between GFP expression level and transgene copy number, we analyzed the skin fibroblasts from SNU-F1-1 and fetal fibroblasts derived from the fetus (SNU-F1-2) of another pregnant transgenic animal, SNU-PB-2 [5]. SNU-PB-2 became pregnant but was gravely injured by other cattle and had to be euthanized. Fluorescent signal was confirmed in the recovered uterus, ovary and oocytes of SNU-PB-2 (Additional file 5: Figure S4). NGS analysis of the genome of SNU-F1-2 identified six transgene integration sites and no genomic instability (Additional file 6: Table S1 and Additional file 7: Figure S5). Both SNU-F1-1 and SNU-F1-2 were generated from a single embryo and there was no mosaicism. As expected, cells from SNU-F1-2 (six copies) showed an approximately 2.2-fold higher expression level of GFP compared to cells from SNU-F1-1 (three copies) (Fig. 6). This result confirms previous work showing that transgene copy number is an important factor to determine the level of transgenic protein expression in transgenic cattle.

\section{Discussion}

Transgenesis in cattle shows great promise for providing insights into basic embryogenesis and disease mechanisms (through the creation of disease models), as well

Table 2 Pattern of SNPs and INDELs from parental DNA

\begin{tabular}{|c|c|c|c|c|c|c|c|}
\hline \multirow{2}{*}{ Father } & \multirow{2}{*}{ Mother } & \multicolumn{3}{|c|}{ SNPs } & \multicolumn{3}{|c|}{ INDELS } \\
\hline & & RefHom $^{1}$ & Hetero $^{2}$ & AltHom $^{3}$ & RefHom & Hetero & AltHom \\
\hline RefHom & RefHom & 0 & 120 & 2 & 0 & 7 & 0 \\
\hline RefHom & Hetero & 123,614 & 93,583 & 6 & 10,537 & 8,154 & 1 \\
\hline RefHom & AltHom & 0 & 11,903 & 3 & 0 & 809 & 0 \\
\hline Hetero & RefHom & 113,234 & 75,948 & 0 & 9,609 & 6,448 & 0 \\
\hline Hetero & Hetero & 112,916 & 227,992 & 117,673 & 9,634 & 20,491 & 9,905 \\
\hline Hetero & AltHom & 0 & 28,758 & 43,359 & 0 & 1,984 & 2,994 \\
\hline AltHom & RefHom & 5 & 12,384 & 0 & 0 & 858 & 0 \\
\hline AltHom & Hetero & 2 & 37,357 & 499 & 0 & 2,738 & 3517 \\
\hline AltHom & AltHom & 0 & 3 & 103,686 & 0 & 0 & 9,418 \\
\hline
\end{tabular}

${ }^{1}$ Homozygous reference genotype

${ }^{2}$ Heterozygous genotype

${ }^{3}$ Homozygous altered genotype

Yellow box: heterozygous de novo mutation not detected in parental DNA

Red box: homozygous de novo mutation not detected in parental DNA 


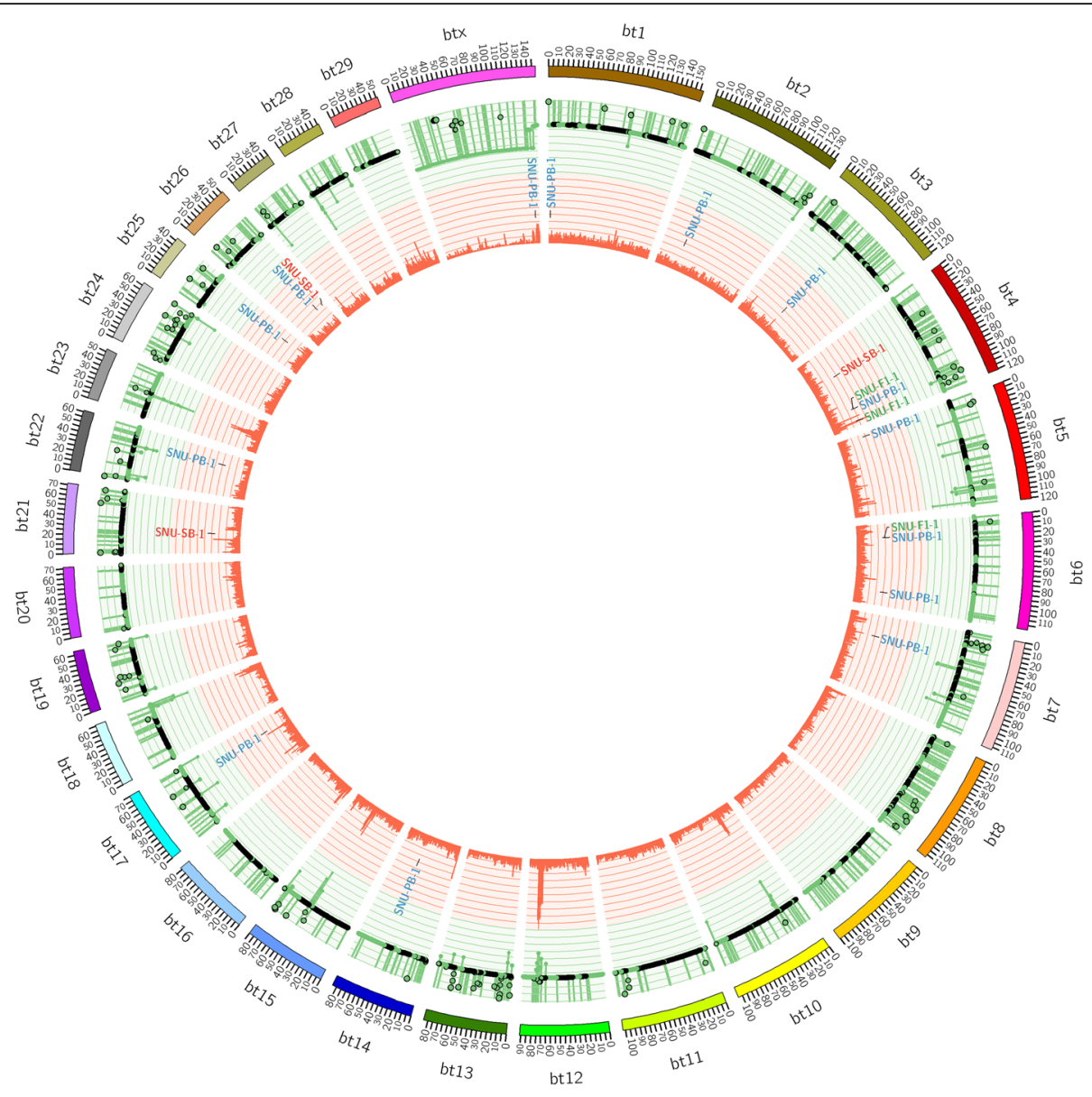

Fig. 5 Overview of genomic variation in SNU-F1-1. Reference chromosomes from bt1 to btX are denoted by colored boxes at the outer edge. Plots denoting copy number variation (CNV; black dot plots in the green area), coverage (green line plot in the green area) and SNP density (orange histogram in orange area) for the SNU-F1-1 genome are shown for each $10 \mathrm{~kb}$ window

as clear potential for protein production and isolation through using cattle as bioreactors. Thus it has long been of interest as a biotechnology in agricultural and veterinary science. However, its progress has been hampered at the practical level by a low efficiency of gene delivery, abnormal reprogramming in cloned embryos and a low success rate for obtaining cloned transgenic offspring with frequent complications (i.e., early embryonic loss and sudden death) $[25,26]$.

Transposon systems have been extensively applied when generating transgenic animal models and have been successfully utilized in zebrafish [27], mice [28], rats [29], pigs [30] and cattle [7]. Moreover, previous studies have verified germline transmission when using transposons in transgenic zebrafish [31], rodents [32, 33] and pigs $[6,11]$. As cattle have a long gestational term and time to puberty, there has only been one previous study, which used lentivirus-mediated transgenesis, to investigate germline transmission in transgenic cattle [34]. In the present study, we have demonstrated that both female and male transgenic cattle with multi-copy integration of transposon-derived transgenes can reach maturity without health issues and we have verified germline transmission through the inheritance of their transgenes by the $\mathrm{F} 1$ generation.

Due to the cut-and-paste action of DNA transposons, it has long been presumed that transposon-mediated transgenesis could create additional genomic instability $[1,35]$. However, many studies have demonstrated that transposons can be used safely and without inducing genomic instability [5, 6, 29, 36-38]. Additionally, in our previous report, all transgenes inserted using either SB or PB in multiple cattle were found to be integrated into non-coding regions. Consistent with previous reports [38], there was no significant difference between the SNU-F1-1 offspring and its parents in terms of SNP, $\mathrm{CNV}$, structural variation (SV) or telomere length as assessed by NGS analysis (Fig. 5). Transgene integration sites were identified in three loci in SNU-F1-1 via NGS analysis (Table 1), and were determined to be only from the paternal genome (SNU-PB-1). As the transgenes integrated into the maternal genome (SNU-SB-1) were 


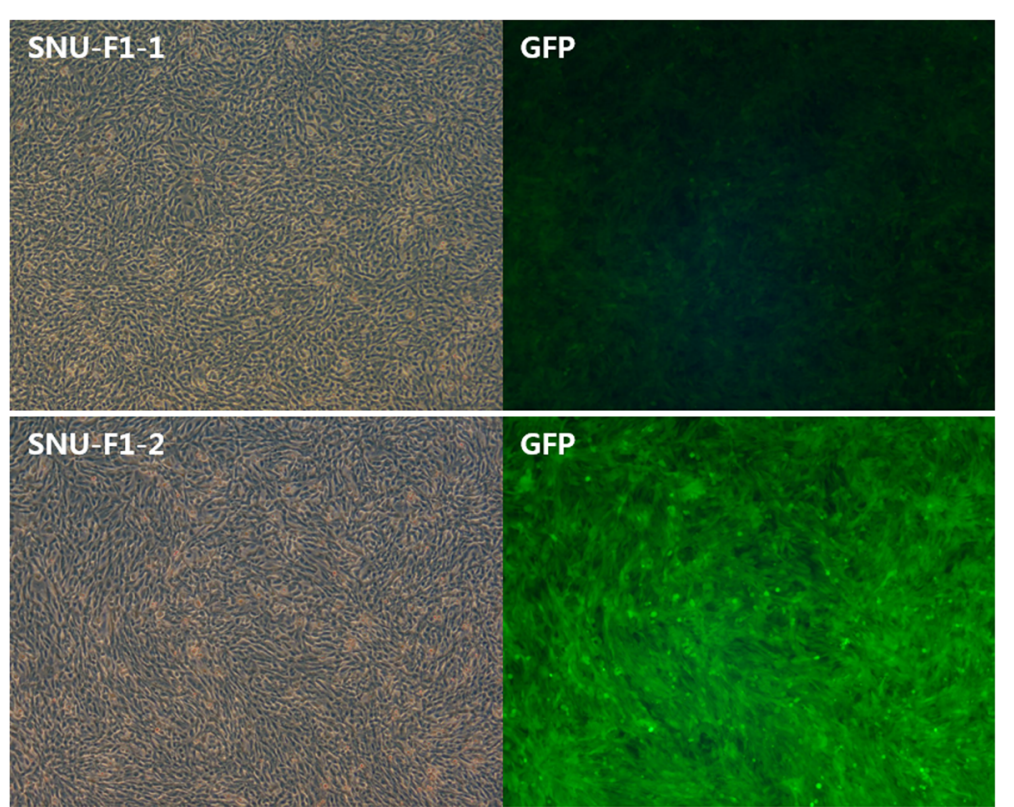

Fig. 6 Expression level of GFP in cells from SNU-F1-1 and SNU-F1-2. Brightfield and fluorescent images of cells from SNU-F1-1 (upper) and SNUF1-2 (lower). GFP: GFP field

heterozygous, it is possible that, following haploid cell generation, an oocyte lacking transgenes was fertilized by the sperm containing the GFP transgenes. As in our previous report, we found that the transgenes in the offspring (SNU-F1-1) were integrated into sites bearing the PB preferred integration sequence, "TTAA" (Additional file 4: Figure S3) [5]. Two integration sites were identical to those seen in SNU-PB-1. One site was not identified in our previous report investigating integration sites in the paternal genome, possibly because that analysis was performed on material from the blood [5] and did not assess integration sites in other organs, such as the testes.

This report also demonstrates that transgenic cattle can act as potential bioreactors for the secretion of exogenous proteins in milk [39-41], as we identified the production of YFP in milk from SNU-SB-1 (Additional file 3: Figure S2). Further, SNU-F1-1 was fed the YFPcontaining milk from SNU-SB-1 for 7 months until weaning. We note that even though the calf consumed milk containing fluorescent proteins for a long period, it has not shown any health issues to date, and so conclude that consumption of transgenic fluorescent protein does not affect health.

The position of the transgene integration site within the genome determines its level of expression or silencing, an effect illustrated by the mosaicism seen during germline transmission in transgenic mice derived from viralmediated gene transfer [42-45], and so the copy number becomes an important determinant of the expression of the transgene. This is a particularly important consideration in transposon-derived transgenic cattle, given their potential use as bioreactor models. Of the cattle derived from germline-transmission of the transgene, SNU-F1-1 and SNU-F1-2 have three and six copies of integrated transgenes, respectively; this allowed an indirect analysis of the relationship between copy number and expression levels in these animals. Although only two samples, we note that the expression level of the transgene in SNU-F1-2 was almost 2.2-fold higher than in SNU-F1-1, indicating that in this case transposon-mediated integration was not affected by silencing or mosaicism.

Our analysis of transgene expression levels in SNU-F1-1 and SNU-F1-2 suggest that the sites of integration in these animals are valuable positions for targeted genome engineering, as we have not observed effects relating to neighboring gene expression or silencing. Therefore, the transgene integration sites identified in SNU-F1-1 will be used for targeted gene expression studies of site-specific knock-in (KI) transgenesis using the CRISPR/Cas9 system. As a proof of principle for this approach, SNU-F1-1 cells were transfected with a single guide RNA targeting GFP, the Cas9 enzyme and a KI donor template that includes RFP and puromycin resistance transgenes between the homology arms. RFP signal was detected in cells following transfection, suggesting effective targeting by CRISPR/ Cas9 (Additional file 8: Figure S6). In addition, we will also investigate whether the transgenes of the $\mathrm{F} 1$ generation will again be transmitted to the next generation (F2) and whether the integration sites are altered or remain stable for future generations.

A further issue that we have attempted to address in this study is whether long-term, stable expression of the 
fluorescent protein in our transgenic cattle (SNU-SB-1, SNU-PB-1, and SNU-F1-1) causes health problems, as GFP has the potential for immunogenicity and cytotoxicity [46]. Female SNU-SB-1, male SNU-PB-1 and F1 calf SNU-F1-1 (male) have all reached maturity at ages of 50, 43 and 19 months old, respectively, and have no general health problems (feeding, growth, body weight, urination or defecation). As further evidence of their health there were no significant abnormalities detected by regular blood analysis (Fig. 2, Additional file 1). These results indicate that transposon-mediated multi-gene integration into the bovine genome and continuous transgene expression does not adversely affect health, for example in organ growth or function. Some RBC, hemoglobin, and hematocrit values in SNU-F1-1 were outside of the reference range (Fig. 2). As previously noted [47], this is most likely due to the age at which blood was collected from the calf, namely at 2 and 10 weeks old. As the genetic variants in SNU-SB-1 and SNU-PB-1 are not significantly different from wild-type cattle, as shown in a previous NGS analysis [5], and the genomic stability (SNP, INDEL and telomere length, etc.) of SNU-F1-1 was not significantly affected (Fig. 5), it is perhaps unsurprising that the cattle generated are healthy. We will continue to monitor their longevity and health status. To our knowledge, this is the first report of transposonderived transgenic cattle surviving for this length of time without any health issues.

\section{Conclusions}

In this study we have demonstrated that multi-copy transgenic cattle derived using the SB and PB systems have survived for more than 3 years without any health issues, and we show for the first time that their transgenes were stably transmitted through the germline to the next generation. The transgenic calf derived through germline transmission has reached maturity at over 19 months old and is healthy, with no significant abnormalities detected by analyses of blood parameters and genomic stability. In conclusion, our study provides valuable data about the safety and long-term expression of transgenes in cattle using transposon-mediated gene modification, and its utility in applications such as exogenous protein expression.

\section{Additional files}

Additional file 1: Raw data of blood analysis from transgenic cattle. (XLSX $15 \mathrm{~kb}$ )

Additional file 2: Figure S1. SNU-F1-1 lacks the YFP transgene, as demonstrated by PCR analysis of genomic DNA from SNU-F1-1. PCR was performed using YFP-specific primers. P/C, positive control (SB-CA-YFP vector); WT, genomic DNA from wild type cattle; Tg:SNU-SB-1, genomic DNA from the blood of SNU-SB-1; Tg:SNU-F1-1, genomic DNA from the blood of SNU-F1-1; N/C, negative control (nuclease-free water). (PNG 99 kb)
Additional file 3: Figure S2. Detection of the expression of YFP in milk from SNU-SB-1 by confocal microscopy. Images of milk from wild type cattle (left) and SNU-SB-1 (right) taken using a high-throughput confocal microscope. YFP: YFP field. (PNG 1685 kb)

Additional file 4: Figure S3. 5' junction sequence analysis of all integration sites in SNU-F1-1. Sequences showing the genome-totransposon junctions in the genome of SNU-F1-1 and the integration of transgenes at TTAA sites. (PNG $465 \mathrm{~kb}$ )

Additional file 5: Figure S4. Germline transmission of GFP expression in uterus, ovary and oocytes from SNU-PB-2. Fluorescent microscope images of GFP expression in: a) uterus from SNU-PB-2, b) ovaries (WT, left; SNU-PB-2, right and arrow) and c) oocytes and cumulus cells from SNUPB-2. (PNG $1557 \mathrm{~kb}$ )

Additional file 6: Table S1. All transgene integration sites in SNU-F1-2. (DOCX $24 \mathrm{~kb}$ )

Additional file 7: Figure S5. Overview of genomic variation in SNU-F1-2. Reference chromosomes from bt 1 to btX are denoted by colored boxes at the outer edge. Plots denoting copy number variation (CNV; black dot plots in the green area), coverage (green line plot in the green area) and SNP density (orange histogram in orange area) for the SNU-F1-2 genome are shown for each $10 \mathrm{~kb}$ window. (PNG $1244 \mathrm{~kb}$ )

Additional file 8: Figure S6. CRISPR/Cas9-mediated KI in SNU-F1-1 cells. (a) Schematic of CRISPR/Cas9-mediated KI of the donor construct. (b) SNU-F1-1 cells were co-transfected with the donor plasmid, Cas9 and sgRNA targeting GFP. The detection of RFP signal and loss of GFP signal in these cells suggests that CRISPR/Cas9-mediated homology directed repair has occurred. pCAG: CAGGS promoter; HA: homology arm; BF: brightfield; GFP: GFP field; RFP: RFP field. (PNG 491 kb)

\section{Abbreviations}

ALT: Alanine transaminase; AST: Aspartate transaminase; BUN: Blood urea nitrogen; CNV: Copy number variation; COC: Cumulus-oocyte complex; GFP: Green fluorescent protein; INDEL: Insertion and deletion; IVF: In vitro fertilization; LTR: Long terminal repeat region; NGS: Next-generation sequencing; PB: PiggyBac; RBC: Red blood cells; RFP: Red fluorescent protein; SB: Sleeping Beauty; SNV: Single nucleotide variant; SV: Structural variation; WBC: White blood cells; YFP: Yellow fluorescent protein

\section{Acknowledgements}

We would like to thank Ji-Hyun Park for performing essential work prior to the commencement of the study. We also thank the members of the G. Jang lab for their valuable comments.

\section{Funding}

This study was supported financially by the Research Institute of Veterinary Science, the Creative Veterinary Graduate program of the Brain Korea 21 Program for Leading Universities \& Students (BK21 PLUS), the Korea Institute of Planning and Evaluation for Technology in Food, Agriculture and Forestry (\# 109023-05-5-CG000) and the National Research Foundation of Korea (2017R1A2B3004972). The funding bodies were not involved in the experimental design and data collection, analysis or interpretation of data in this study, nor in the writing of the manuscript.

\section{Availability of data and materials}

The raw NGS datasets used in this study are available at NCBI (BIO-project number: PRJNA438569; SRA: SRR6849472, SRR6849471, SRR6849470; https:// www.ncbi.n/m.nih.gov/bioproject/PRJNA438569).

\section{Authors' contributions}

S-YY wrote the manuscript, generated DNA constructs, performed cell transfection, cell culture and data collection; S-JL performed microinjections, cultured embryos and collected data; S-GP performed bioinformatics analyses and wrote the manuscript; I-GS performed bioinformatics analyses and interpreted the data; S-EH cultured cells and performed PCR; W-JC performed microinjections and cultured embryos; H-SK performed microinjections, cultured embryos and collected data; $\mathrm{H}$-JK performed in vitro maturation of oocytes, performed microinjections and collected data; S-HB transferred embryos, performed animal husbandry and analyzed transgenic cattle; J-HL transferred embryos, performed animal husbandry 
and analyzed transgenic cattle; J-YM transferred embryos, performed animal husbandry and analyzed transgenic cattle; W-SL transferred embryos and performed animal husbandry; J-HL performed in vitro fertilization and in vitro culturing and collected embryos; C-IL performed embryo microinjection and in vitro fertilization; S-JK performed the bioinformatics analyses with I-GS and S-GP, and conceived and designed the study; GJ provided overall supervision, designed the study, analyzed data with S-YY and wrote the manuscript. All authors reviewed the manuscript before submission. All authors read and approved the final manuscript.

\section{Ethics approval}

All experiments with live animals were performed in accordance with the relevant laws and institutional guidelines of Seoul National University and Seoul Milk Coop [5]. The experiment was approved by the institutional committee of the Seoul Milk Coop and the national committee of Living Modified Organism (LMO) development and experimentation at the Ministry of Science and Information \& Communications Technology (ICT) (\#LMR 16-29; https://www.Imosafety.or.kr/). All transgenic cattle [5] were registered as being for academic purposes at the local district office and monitored in a permitted area (\#LML16-305) by the Ministry of Science and ICT according to the regulations and guidelines of South Korea's national animal husbandry law and LMO regulations. The field studies, including collection of specimens and veterinary care, were conducted by a veterinarian.

\section{Competing interests}

The authors declare no competing financial interests.

\section{Publisher's Note}

Springer Nature remains neutral with regard to jurisdictional claims in published maps and institutional affiliations.

\section{Author details}

'Department of Theriogenology, College of Veterinary Medicine and the Research Institute of Veterinary Science, Seoul National University, \#631 Building 85, Gwanak-ro, Gwanak-gu, Seoul 08826, Republic of Korea. ${ }^{2}$ Embryo Research Center, Seoul Milk Coop, Gyeonggi-do 12528, Republic of Korea. ${ }^{3}$ Bioinformatics Team, Theragen Etex Bio Institute, Advanced Institutes of Convergence Technology, Kwanggyo Technovalley, Suwon 16229, Republic of Korea. ${ }^{4}$ Emergence Center for Food-Medicine Personalized Therapy System, Advanced Institutes of Convergence Technology, Seoul National University, Gyeonggi-do 16229, Republic of Korea.

\section{Received: 13 October 2017 Accepted: 4 May 2018}

\section{Published online: 23 May 2018}

\section{References}

1. VandenDriessche T, Ivics Z, Izsvak Z, Chuah MK. Emerging potential of transposons for gene therapy and generation of induced pluripotent stem cells. Blood. 2009;114:1461-8.

2. Aronovich EL, Mclvor RS, Hackett PB. The sleeping beauty transposon system: a non-viral vector for gene therapy. Hum Mol Genet. 2011;20:R14-20.

3. Kebriaei $P$, Izsvak Z, Narayanavari SA, Singh H, Ivics Z. Gene therapy with the sleeping beauty transposon system. Trends Genet. 2017;33:852-70.

4. Woodard LE, Wilson MH. piggyBac-ing models and new therapeutic strategies. Trends Biotechnol. 2015;33:525-33.

5. Yum SY, Lee SJ, Kim HM, Choi WJ, Park JH, Lee WW, Kim HS, Kim HJ, Bae $\mathrm{SH}$, Lee $\mathrm{JH}$, et al. Efficient generation of transgenic cattle using the DNA transposon and their analysis by next-generation sequencing. Sci Rep. 2016; 6:27185.

6. Garrels W, Mates L, Holler S, Dalda A, Taylor U, Petersen B, Niemann H, Izsvak Z, Ivics Z, Kues WA. Germline transgenic pigs by sleeping beauty transposition in porcine zygotes and targeted integration in the pig genome. PLoS One. 2011;6:e23573.

7. Garrels W, Talluri TR, Apfelbaum R, Carratala YP, Bosch P, Potzsch K, Grueso E, Ivics Z, Kues WA. One-step multiplex Transgenesis via sleeping beauty transposition in cattle. Sci Rep. 2016;6:21953.

8. Deng S, Li G, Yu K, Tian X, Wang F, Li W, Jiang W, Ji P, Han H, Fu J, et al. RNAi combining sleeping beauty transposon system inhibits ex vivo expression of foot-and-mouth disease virus VP1 in transgenic sheep cells. Sci Rep. 2017;7:10065.
9. Bai DP, Yang MM, Qu L, Chen YL. Generation of a transgenic cashmere goat using the piggyBac transposition system. Theriogenology. 2017;93:1-6.

10. Carlson DF, Garbe JR, Tan W, Martin MJ, Dobrinsky JR, Hackett PB, Clark KJ, Fahrenkrug SC. Strategies for selection marker-free swine transgenesis using the sleeping beauty transposon system. Transgenic Res. 2011;20:1125-37.

11. Ivics Z, Garrels W, Mates L, Yau TY, Bashir S, Zidek V, Landa V, Geurts A, Pravenec M, Rulicke T, et al. Germline transgenesis in pigs by cytoplasmic microinjection of sleeping beauty transposons. Nat Protoc. 2014;9:810-27.

12. Hallet B, Sherratt DJ. Transposition and site-specific recombination: adapting DNA cut-and-paste mechanisms to a variety of genetic rearrangements. FEMS Microbiol Rev. 1997;21:157-78.

13. Gogol-Doring A, Ammar I, Gupta S, Bunse M, Miskey C, Chen W, Uckert W, Schulz TF, Izsvak Z, Ivics Z. Genome-wide profiling reveals remarkable parallels between insertion site selection properties of the MLV retrovirus and the piggyBac transposon in primary human CD4+ T cells. Mol Ther. 2016:24:592-606.

14. Hackett PB, Largaespada DA, Switzer KC, Cooper LJ. Evaluating risks of insertional mutagenesis by DNA transposons in gene therapy. Transl Res. 2013;161:265-83.

15. Williams DA. Sleeping beauty vector system moves toward human trials in the United States. Mol Ther. 2008;16:1515-6.

16. Kebriaei $P$, Singh $H$, Huls MH, Figliola MJ, Bassett R, Olivares S, Jena B, Dawson MJ, Kumaresan PR, Su S, et al. Phase I trials using sleeping beauty to generate CD19-specific CAR T cells. J Clin Invest. 2016;126:3363-76.

17. Kebriaei $P$, Huls $H$, Jena B, Munsell M, Jackson R, Lee DA, Hackett PB, Rondon G, Shpall E, Champlin RE, et al. Infusing CD19-directed T cells to augment disease control in patients undergoing autologous hematopoietic stem-cell transplantation for advanced B-lymphoid malignancies. Hum Gene Ther. 2012:23:444-50.

18. Moriarity BS, Largaespada DA. Sleeping beauty transposon insertional mutagenesis based mouse models for cancer gene discovery. Curr Opin Genet Dev. 2015;30:66-72.

19. Machado GM, Carvalho JO, Filho ES, Caixeta ES, Franco MM, Rumpf R, Dode MA. Effect of Percoll volume, duration and force of centrifugation, on in vitro production and sex ratio of bovine embryos. Theriogenology. 2009;71:1289-97.

20. Lim KT, Jang G, Ko KH, Lee WW, Park HJ, Kim JJ, Lee SH, Hwang WS, Lee BC, Kang SK. Improved in vitro bovine embryo development and increased efficiency in producing viable calves using defined media. Theriogenology. 2007;67:293-302.

21. Zhang R, Yin Y, Zhang Y, Li K, Zhu H, Gong Q, Wang J, Hu X, Li N. Molecular characterization of transgene integration by next-generation sequencing in transgenic cattle. PLoS One. 2012;7:e50348.

22. Roach JC, Glusman G, Smit AF, Huff CD, Hubley R, Shannon PT, Rowen L, Pant KP, Goodman N, Bamshad M, et al. Analysis of genetic inheritance in a family quartet by whole-genome sequencing. Science. 2010;328:636-9.

23. Soboleski MR, Oaks J, Halford WP. Green fluorescent protein is a quantitative reporter of gene expression in individual eukaryotic cells. FASEB J. 2005;19:440-2.

24. Fortinea N, Trieu-Cuot P, Gaillot O, Pellegrini E, Berche P, Gaillard JL. Optimization of green fluorescent protein expression vectors for in vitro and in vivo detection of listeria monocytogenes. Res Microbiol. 2000;151:353-60.

25. Tamada $\mathrm{H}$, Kikyo N. Nuclear reprogramming in mammalian somatic cell nuclear cloning. Cytogenet Genome Res. 2004;105:285-91.

26. Rideout WM 3rd, Eggan K, Jaenisch R. Nuclear cloning and epigenetic reprogramming of the genome. Science. 2001;293:1093-8.

27. Wu SC, Meir YJ, Coates CJ, Handler AM, Pelczar P, Moisyadi S, Kaminski JM. piggyBac is a flexible and highly active transposon as compared to sleeping beauty, Tol2, and Mos1 in mammalian cells. Proc Natl Acad Sci U S A. 2006: 103:15008-13

28. Ding S, Wu X, Li G, Han M, Zhuang Y, Xu T. Efficient transposition of the piggyBac (PB) transposon in mammalian cells and mice. Cell. 2005;122:473-83.

29. Li T, Shuai L, Mao J, Wang X, Wang M, Zhang X, Wang L, Li Y, Li W, Zhou Q. Efficient production of fluorescent transgenic rats using the piggyBac transposon. Sci Rep. 2016;6:33225.

30. Jakobsen JE, Li J, Kragh PM, Moldt B, Lin L, Liu Y, Schmidt M, Winther KD, Schyth BD, Holm IE, et al. Pig transgenesis by sleeping beauty DNA transposition. Transgenic Res. 2011:20:533-45.

31. Balciunas D, Davidson AE, Sivasubbu S, Hermanson SB, Welle Z, Ekker SC. Enhancer trapping in zebrafish using the sleeping beauty transposon. BMC Genomics. 2004:5:62.

32. Ivics Z, Mates L, Yau TY, Landa V, Zidek V, Bashir S, Hoffmann OI, Hiripi L, Garrels W, Kues WA, et al. Germline transgenesis in rodents by pronuclear microinjection of sleeping beauty transposons. Nat Protoc. 2014;9:773-93. 
33. Garcia Diaz Al, Moyon B, Coan PM, Alfazema N, Venda L, Woollard K, Aitman T. New Wistar Kyoto and spontaneously hypertensive rat transgenic models with ubiquitous expression of green fluorescent protein. Dis Model Mech. 2016;9:463-71.

34. Reichenbach M, Lim T, Reichenbach HD, Guengoer T, Habermann FA, Matthiesen M, Hofmann A, Weber F, Zerbe H, Grupp T, et al. Germ-line transmission of lentiviral PGK-EGFP integrants in transgenic cattle: new perspectives for experimental embryology. Transgenic Res. 2010;19:549-56.

35. Saha S, Woodard LE, Charron EM, Welch RC, Rooney CM, Wilson MH. Evaluating the potential for undesired genomic effects of the piggyBac transposon system in human cells. Nucleic Acids Res. 2015;43:1770-82.

36. Katter K, Geurts AM, Hoffmann O, Mates L, Landa V, Hiripi L, Moreno C, Lazar J, Bashir S, Zidek V, et al. Transposon-mediated transgenesis, transgenic rescue, and tissue-specific gene expression in rodents and rabbits. FASEB J. 2013;27:930-41.

37. Fischer SE, Wienholds E, Plasterk RH. Regulated transposition of a fish transposon in the mouse germ line. Proc Natl Acad Sci U S A. 2001;98:6759-64.

38. Clark KJ, Urban MD, Skuster KJ, Ekker SC. Transgenic zebrafish using transposable elements. Methods Cell Biol. 2011;104:137-49.

39. Kerekes A, Hoffmann Ol, Iski G, Liptak N, Gocza E, Kues WA, Bosze Z, Hiripi L. Secretion of a recombinant protein without a signal peptide by the exocrine glands of transgenic rabbits. PLoS One. 2017;12:e0187214.

40. Mukherjee A, Garrels W, Talluri TR, Tiedemann D, Bosze Z, Ivics Z, Kues WA. Expression of active fluorophore proteins in the milk of transgenic pigs bypassing the secretory pathway. Sci Rep. 2016;6:24464.

41. Yang B, Wang J, Tang B, Liu Y, Guo C, Yang P, Yu T, Li R, Zhao J, Zhang L, et al. Characterization of bioactive recombinant human lysozyme expressed in milk of cloned transgenic cattle. PLoS One. 2011;6:e17593.

42. Bryda EC, Pearson M, Agca Y, Bauer BA. Method for detection and identification of multiple chromosomal integration sites in transgenic animals created with lentivirus. BioTechniques. 2006;41:715-9.

43. Park F. Lentiviral vectors: are they the future of animal transgenesis? Physiol Genomics. 2007:31:159-73.

44. Jahner $D$, Jaenisch R. Integration of Moloney leukaemia virus into the germ line of mice: correlation between site of integration and virus activation Nature. 1980;287:456-8.

45. Garrick D, Fiering S, Martin DI, Whitelaw E. Repeat-induced gene silencing in mammals. Nat Genet. 1998;18:56-9.

46. Ansari AM, Ahmed AK, Matsangos AE, Lay F, Born LJ, Marti G, Harmon JW, Sun Z. Cellular GFP toxicity and immunogenicity: potential confounders in in vivo cell tracking experiments. Stem Cell Rev. 2016;12:553-9.

47. Brun-Hansen $\mathrm{HC}$, Kampen AH, Lund A. Hematologic values in calves during the first 6 months of life. Vet Clin Pathol. 2006;35:182-7.

\section{Ready to submit your research? Choose BMC and benefit from:}

- fast, convenient online submission

- thorough peer review by experienced researchers in your field

- rapid publication on acceptance

- support for research data, including large and complex data types

- gold Open Access which fosters wider collaboration and increased citations - maximum visibility for your research: over $100 \mathrm{M}$ website views per year

At BMC, research is always in progress.

Learn more biomedcentral.com/submissions 